\title{
Thapsigargin Induces Apoptosis by Impairing Cytoskeleton Dynamics in Human Lung Adenocarcinoma Cells
}

\author{
Fei Wang, ${ }^{1}$ Da-zhong Liu, ${ }^{1}$ Hao Xu, ${ }^{1}$ Yi Li, ${ }^{1}$ Wei Wang, ${ }^{1}$ Bai-lu Liu, ${ }^{2}$ and Lin-you Zhang \\ ${ }^{1}$ Department of Thoracic Surgery, The Second Affiliated Hospital of Harbin Medical University, No. 246 Xuefu, \\ Nangang District, Harbin 150086, China \\ ${ }^{2}$ Department of Computerized Tomography, The Second Affiliated Hospital of Harbin Medical University, No. 246 Xuefu, \\ Nangang District, Harbin 150086, China \\ Correspondence should be addressed to Bai-lu Liu; liubailu2008@126.com and Lin-you Zhang; zhanglyhxj@163.com
}

Received 30 August 2013; Accepted 10 November 2013; Published 28 January 2014

Academic Editors: T. Hida, T. Liu, and A. D. L. Sihoe

Copyright (C) 2014 Fei Wang et al. This is an open access article distributed under the Creative Commons Attribution License, which permits unrestricted use, distribution, and reproduction in any medium, provided the original work is properly cited.

\begin{abstract}
The objective of this study was performed to investigate the effects of thapsigargin on apoptosis, actin cytoskeletal dynamics, and actin cytoskeletal proteins in human lung adenocarcinoma cell. Thapsigargin is a specific irreversible inhibitor of ER calciumATPase, which may promote ER stress by depletion of lumenal calcium stores and show potential to induce cell death. The effects of thapsigargin on the apoptosis in A549 cells were assayed by Hoechst staining. Moreover, the F-actin staining by Rhodaminephalloidin and RhoA antibody for cytoskeleton organizations were applied to A549 cells. To confirm the impairment of cytoskeletal dynamics treated with thapsigargin, western blots were applied to analyze the protein levels of p-Cofilin-1 (Ser3), Cofilin-1, and pPaxillin (Tyr118), as well as RhoA and pS6 (S240/244). Results suggest that thapsigargin may induce cell death in A549 cells with a time- and dose-dependent manner. The F-actin fibers and RhoA signals are also reduced with a time- and dose-dependent manner by thapsigargin treatment. The phosphorylation forms of Cofilin-1 and paxillin are attenuated by $1 \mu \mathrm{M}$ thapsigargin treatment for $24 \mathrm{~h}$. These alternations may be caused by the inhibition of of mTORC1 activities (indicated by pS6 (Ser240/244)) and RhoA pathways after thapsigargin treatment. The present findings highlight important roles of calcium entry in cytoskeleton organization and apoptosis in human lung adenocarcinoma cells and will help to set a stage to the clinical treatment of cancer cell metastasis.
\end{abstract}

\section{Introduction}

Cytoskeleton is required for many biological processes, such as embryonic morphogenesis, immune surveillance, tissue repair, and regeneration. Aberrant regulation of cytoskeleton dynamics drives progression of cancer invasion and metastasis $[1,2]$. Cancer cell metastasis is a multistage process involving invasion into surrounding tissue, intravasation, transit in the blood or lymph, extravasation, and growth at a new site. Many of these steps require cell motility, which is driven by cycles of actin polymerization and depolymerization [3]. Actin networks consist of branched or linear filaments and regulate many essential cellular processes. The actin cytoskeleton functions in the generation and maintenance of cell morphology and polarity and in endocytosis, intracellular trafficking, contractility, motility, and cell division. The assembly and disassembly of actin filaments, as well as their organizations into functional higher-order networks, are regulated by several actin-binding proteins, many of which are conserved from yeast to human $[4,5]$.

Malignant cancer cells utilize their intrinsic migratory ability to invade adjacent tissues and the vasculature and ultimately to metastasize. The motility of tumor cells is driven by the polymerization of actin monomers into polarized filaments. These filaments, termed F-actin, are in a constant state of flux with new monomers being added [6,7]. One of the proteins identified in F-actin formation is Cofilin1. Cofilin-1 is an actin-depolymerizing factor and regulates actin cytoskeletal reorganization. Phosphorylation of Cofilin1 on Serine-3 is known to block these activities, which is regulated by RhoA GTPases through mTORC1-RhoALimk-Cofilin-1 pathways $[8,9]$. Earlier results have been coupled with intracellular calcium influx and cytoskeletal dynamic in vitro and in vivo. For example, calcium influx 
into rat brain synaptosomes causes the breakdown of Factin under the plasma membrane [10-12]. And inhibition of actin polymerization has a biphasic time-dependent effect on calcium entry, showing an initial potentiation followed by inhibition of calcium entry [13]. Moreover, thapsigargin, a specific irreversible inhibitor of ER calcium-ATPase, has been used to investigate the effect of a disturbed endoplasmic reticulum (ER) calcium homeostasis on different processes of cells, including cytoskeleton dynamics. It is interesting that thapsigargin-induced depletion of ER calcium stores severely inhibits F-actin contents in human monocytic cells [14]. Thus, thapsigargin may prove to be a useful tool for investigating calcium influx and cytoskeleton dynamics underlying cell apoptosis.

Although progress has been made, it is still largely unknown whether the calcium influx couples with cytoskeletal dynamics and affects cell apoptosis, especially in cancer cells. To examine the critical role between calcium signaling, cytoskeleton, and cell apoptosis, we focus on the effect of ectopic entry of calcium influx by thapsigargin on actin cytoskeleton apoptosis in A549 human lung adenocarcinoma cell lines. Our findings demonstrate that thapsigargin treatment may induce cell death in A549 cells in a time- and dose-dependent manner. For ectopic entry of calcium influx being induced thapsigargin, we find that the cytoskeletal dynamics is impaired by thapsigargin treatment, indicated by F-actin staining and biochemical evidence. The effects of thapsigargin on cell death in A549 tumor cells may be mediated by mTORC1-RhoA-Cofilin-1 pathway, because thapsigargin treatment may dramatically inhibit mTORC1 activity and RhoA protein level. Our work will help to understand a novel clue regarding the relationship between calcium signaling, cytoskeleton, and cell apoptosis in A549 tumor cells.

\section{Materials and Methods}

2.1. Chemicals and Materials. The inhibitor of ER calciumATPase thapsigargin was purchased from Sigma-Aldrich (St. Louis, MO, USA). Dulbecco's Modified Essential Medium (DMEM), Fetal Bovine Serum (FBS), and F-actin probes of Rhodamine Phalloidin R415 were purchased from Gibco Invitrogen Corporation (Carlsbad, CA, USA). The Hoechst kit was from Beyotime Biotechnology Co. (Haimen, Jiangsu, China). The following antibodies, anti-p-Cofilin-1, antiCofilin-1, and anti-RhoA were from Santa Cruz Biotechnology (Santa Cruz, CA, USA). The p-Paxillin (Tyr118) and pS6 (Ser240/244) antibodies were from Cell Signaling Technology (Danvers, MA, USA) and anti-GAPDH was from Millipore (Billerica, MA, USA). Other chemicals were of the highest purity available.

2.2. Assays of Cell Culture. A widely used human lung adenocarcinoma cell line A549 was chosen as in vitro experiment system, which was obtained from Shanghai Institute of Cell Biology (Introduced from American Type Culture Collection). A549 cells were derived from a lung adenocarcinoma and widely used to study the amplification process in tumors. In the present study, A549 cells were plated in 6-well plates at $1.0 \times 10^{6}$ cells $/ \mathrm{mL}$. The cells were incubated in Dulbecco's Modified Essential Medium (DMEM) containing 10\% Fetal Bovine Serum (FBS) plus antibiotics for $24 \mathrm{~h}$ in $5 \% \mathrm{CO}_{2}$ at $37^{\circ} \mathrm{C}$.

2.3. Pharmacological Manipulations. For ectopic calcium influx in A549 cells, the final concentrations (1, 100, and $1000 \mathrm{nM})$ of thapsigargin were applied to these cells and then incubated from $6 \mathrm{~h}$ to $24 \mathrm{~h}$. No additives were used as internal controls. After culturing, the cells were harvested for subsequent Hoechst stainings and immunostainings. To further study the role of thapsigargin on cytoskeleton molecules in A549 cells, thapsigargin of $1 \mu \mathrm{M}$ for $6 \mathrm{~h}$ was applied to A549 cells for biochemical examinations.

2.4. Assay of Hoechst Staining. For the preparation of Hoechst staining, A549 cells were plated with $1.0 \times 10^{5}$ cells $/ \mathrm{mL}$ in 6-well plates. After pharmacological manipulations, cells were directly stained with Hoechst kit from Beyotime. The cell counting was carried out through the use of National Institutes of Health software ImageJ, which is available at http://rsbweb.nih.gov.

2.5. Assay of F-Actin Staining and Immunostaining. To detect the F-actin fibers in thapsigargin-treated A549 cells, the Rhodamine Phalloidin R415 probes were applied to stain the F-actin fibers. For the preparation of Rhodamine Phalloidin staining, A549 cells were plated with $1.0 \times 10^{5}$ cells $/ \mathrm{mL}$ in 6 well plates. After thapsigargin-treated at different concentrations from $6 \mathrm{~h}$ to $24 \mathrm{~h}$, cells were fixed with $4 \%$ Paraformaldehyde (PFA) and 4\% sucrose in phosphate-buffered saline for $30 \mathrm{~min}$ and then permeabilized with $0.25 \%$ Triton-X 100 in PBS for another $5 \mathrm{~min}$ at room temperature. Anti-RhoA antibody was used to detect RhoA signals in these cells. After washing extensively, Rhodamine Phalloidin R415 probes were added into these cells, as well as antibodies-Alexa Fluor 594 goat anti-mouse IgG (Invitrogen, Carlsbad, CA). Then after a second washing for three times, the cover slips were mounted onto glass slides with antifade reagent with $4^{\prime}, 6$-diamidino-2phenylindole (DAPI) for nuclear labellings.

2.6. Assay of Western Blots. To extract proteins, cultured A549 cells were sonicated with lysis buffer (PBS with $1 \%$ Triton X-100 and protease inhibitors). The cell lysate supernatants were harvested by centrifugation at $10000 \mathrm{rpm}$ for $10 \mathrm{~min}$ at $4^{\circ} \mathrm{C}$. Protein concentrations of the cell supernatants were evaluated and measured by BCA Protein Assay kit (Thermo Fisher Scientific Inc., Rockford, IL, USA). Equal amounts of the proteins from each extract were separated in a SDS-polyacrylamide gel (12.6\%) with 5\% stacking gel in SDS-Tris-glycine running buffer. The proteins were then transferred electrophoretically using a PVDF membrane by standard procedures. The membranes were then blocked by $5 \%$ nonfat dry milk in PBST (PBS with $0.1 \%$ Tween 20, pH 7.6) for $1 \mathrm{~h}$ at room temperature and probed overnight by proper primary antibodies diluted in PBST at $4^{\circ} \mathrm{C}$. The membranes were rinsed 3 times with PBST and incubated with proper secondary antibodies diluted in PBST for $1 \mathrm{~h}$ at room temperature. Then, the membranes were rinsed another 3 times 

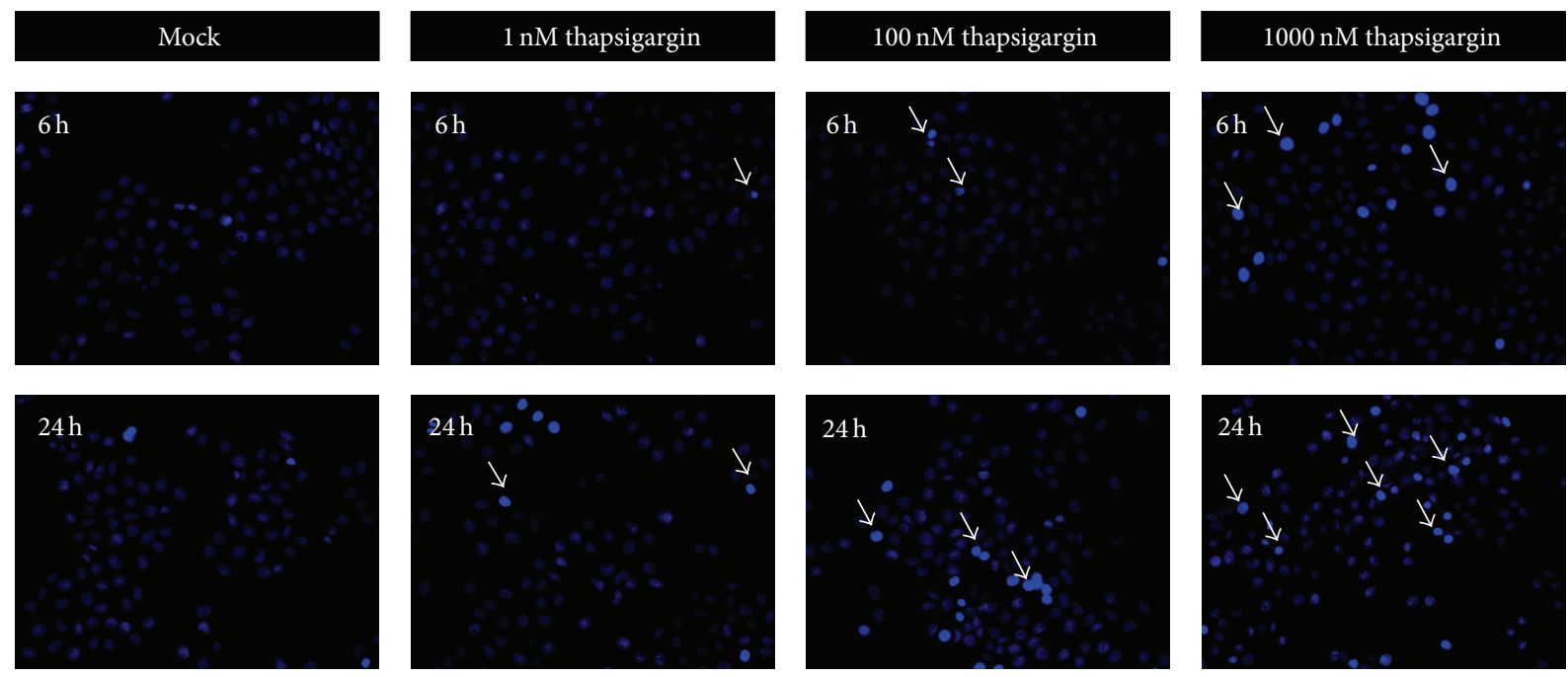

(a)

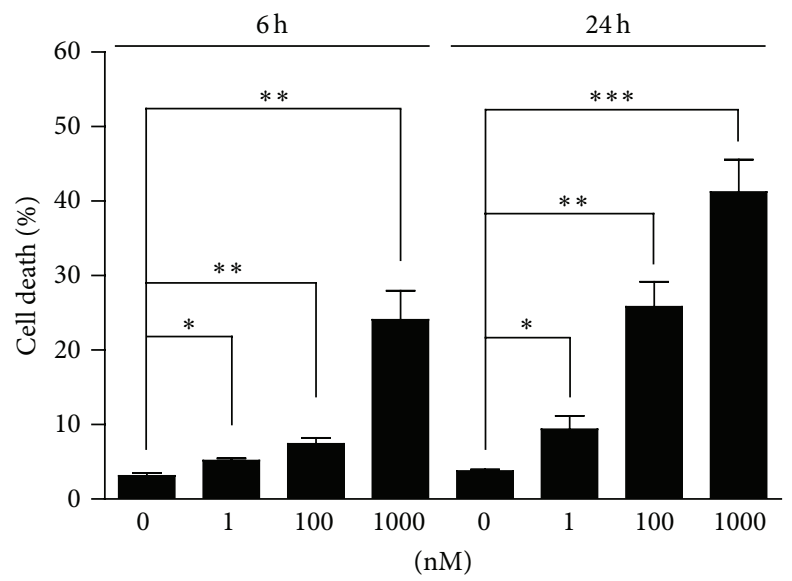

(b)

Figure 1: Thapsigargin induces cell apoptosis in A549 cells. (a) Hoechst staining (blue) showing the increasing of apoptotic A549 cells by thapsigargin $(1 \mathrm{nM}, 100 \mathrm{nM}$, and $1 \mu \mathrm{M})$ treatment for 6 and $24 \mathrm{~h}$ (white arrows). (b) Histograms showing the quantification of the cell death (\%) in A549 cells after thapsigargin treatment. Results are averages of three independent experiments. Data represent mean \pm SEM. ${ }^{*} P<0.05$, ${ }^{* *} P<0.01$, and ${ }^{* * *} P<0.001$.

with PBST at room temperature for $10 \mathrm{~min}$, and proteins were detected by Super Signal enhanced chemiluminescence development (ECL) (Thermo Scientific Pierce) reagent and exposed to films (Kodak). The protein level quantification was carried out by ImageJ.

2.7. Statistical Analysis. All statistical analysis was performed by Image software. Quantitative data were shown in $x^{-} \pm s$ using $t$-tests for comparisons. The values $0.05\left({ }^{*}\right), 0.01\left({ }^{* *}\right)$, and $0.001\left(^{* * *}\right)$ were assumed as the level of significance for the statistic tests carried out.

\section{Results}

3.1. Thapsigargin Induces Cell Apoptosis in A549 Cells. To examine whether the thapsigargin treatment may induce the cell death in A549 cells, we applied Hoechst staining to
A549 cells treated by thapsigargin (Figure 1). The results show that thapsigargin has slight effects on cell death at the final concentration of $1 \mathrm{nM}$ (cell death by $5.2 \%$ ) or $100 \mathrm{nM}$ (by $7.4 \%$ ) for $6 \mathrm{~h}$ treatment (Figure $1(\mathrm{~b})$ ). The percentage of cell death increases significantly to $24.1 \%$ at the concentration of $1 \mu \mathrm{M}$ (Figure 1(b)). To examine whether the effects of thapsigargin on cell death of A549 cells is time-dependent or not, we prolonged the treated time of thapsigargin on A549 cells to $24 \mathrm{~h}$. Our results suggest that the percentages of cell death increase to $9.4 \%$ ( $1 \mathrm{nM}), 25.8 \%(100 \mathrm{nM})$, and $41.2 \%(1 \mu \mathrm{M})$ after thapsigargin treatment (Figure $1(\mathrm{~b}))$. These findings support the notion that thapsigargin may induce cell death in A549 cells in a time- and dose-dependent manner.

3.2. Thapsigargin Impairs Actin Cytoskeleton Organizations in A549 Cells. To study the cellular mechanisms of how thapsigargin induces cell death in A549 cells, we focused 

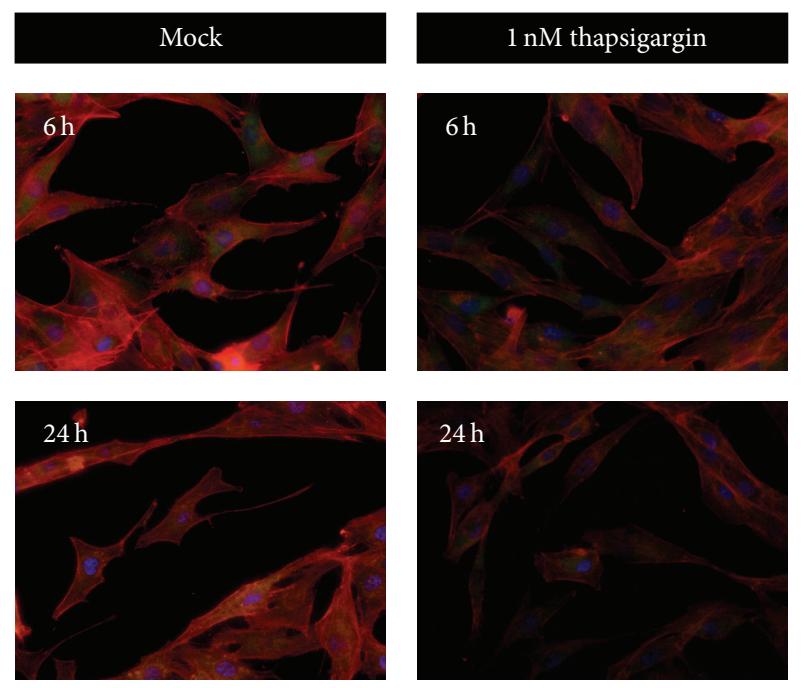
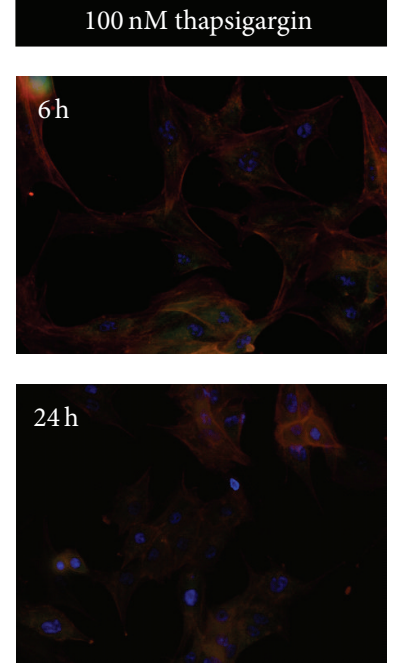

$1000 \mathrm{nM}$ thapsigargin
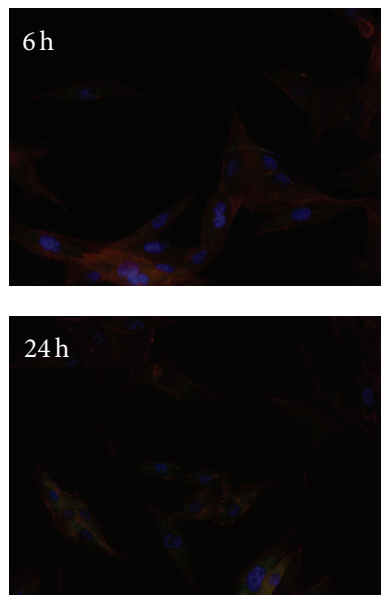

FIGURE 2: Thapsigargin impairs actin cytoskeleton organizations in A549 cells. The reductions of F-actin fibers by thapsigargin (1 $\mathrm{nM}, 100 \mathrm{nM}$, and $1 \mu \mathrm{M}$ for 6 and $24 \mathrm{~h}$ ) treatments were shown by immunostaining. Red fluorescence is indicated by Rhodamine-phalloidin probes, green by RhoA antibody, and blue by DAPI.

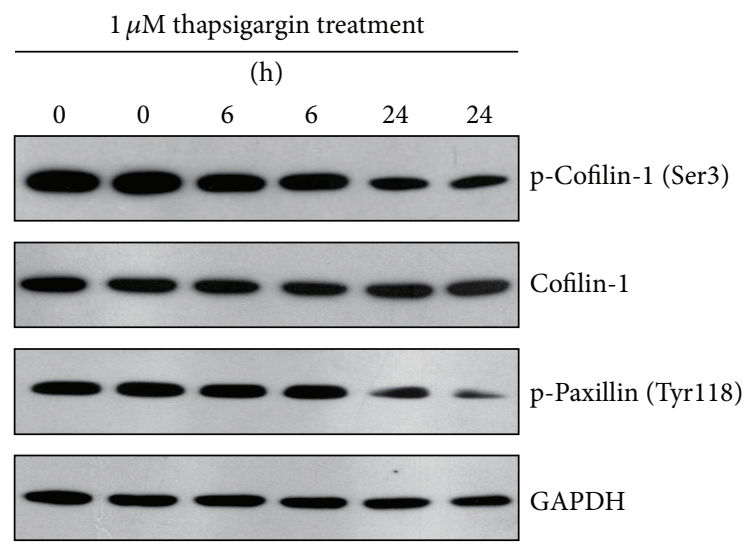

(a)

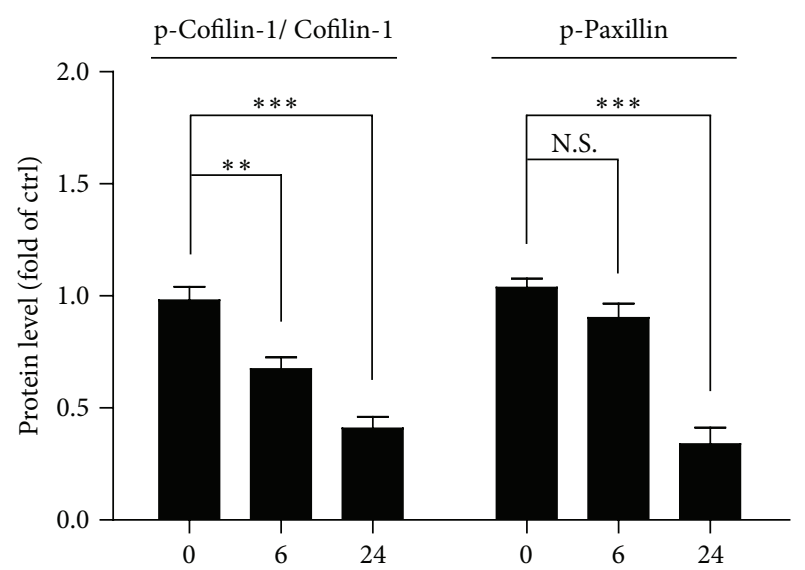

(h)

(b)

FIGURE 3: Thapsigargin disrupts actin cytoskeletal proteins in A549 cells. The reductions of protein levels of p-Cofilin-1 (Ser3) and p-Paxillin (Tyr118) by thapsigargin treatment ( $1 \mu \mathrm{M}$ for 6 and $24 \mathrm{~h}$ ) in A549 cells were shown by Western blots (a) and histograms (b). Notes showed that the protein level of total Cofilin-1 is not affected by thapsigargin treatment. Results are averages of four independent experiments. Data represent mean \pm SEM. ${ }^{* *} P<0.01,{ }^{* * *} P<0.001$, N.S, and no statistical difference.

on the cytoskeletal dynamics, because we noted that A549 cells tended to shrink after thapsigargin treatment (Data not shown). Thus, we carried out F-actin staining by Rhodamine labeled Phalloidin probes in A549 cells. Being consistent with Hoechst stainings, our results show that the F-actin fibers are reduced in a time- and dose-dependent manner after thapsigargin treatment (Figure 2). Moreover, RhoA signals, indicated by the greed-fluorescence, are also reduced after thapsigargin treatment, in parallel with Rhodaminephalloidin signals, while DAPI signals labelled blue indicate the nuclear locations (Figure 2). The parallel reduction of Factin and RhoA signals by thapsigargin treatment confirms that thapsigargin may impair the cytoskeleton dynamics and organizations.
3.3. Thapsigargin Disrupts Actin Cytoskeletal Proteins in A549 Cells. To confirm the impairment of cytoskeletal dynamics in thapsigargin treated A549 cells, we examined the cellular pathways regulating F-actin organizations. By western blots, we show that Cofilin-1 phosphorylations are reduced after thapsigargin treatment, while the total protein levels of Cofilin-1 are not altered (Figure 3(a)). The ratio of p-Cofilin-1 to Cofilin-1 reduces to $67.4 \%$ after thapsigargin treatment of $1 \mu \mathrm{M}$ for $6 \mathrm{~h}$, and to $40.9 \%$ after $1 \mu \mathrm{M}$ for $24 \mathrm{~h}$ (Figure $3(\mathrm{~b})$ ). Consistently, the phosphorylation of paxillin is also reduced to $33.9 \%$ after $1 \mu \mathrm{M}$ thapsigargin treatment for $24 \mathrm{~h}$ (Figures $3(\mathrm{a})$ and 3(b)). It is of note that the protein level of p-Paxillin is not altered with statistical difference after $1 \mu \mathrm{M}$ thapsigargin treatment for $6 \mathrm{~h}$ (Figures 3(a) and 3(b)). This may be due 
$1 \mu \mathrm{M}$ thapsigargin treatment

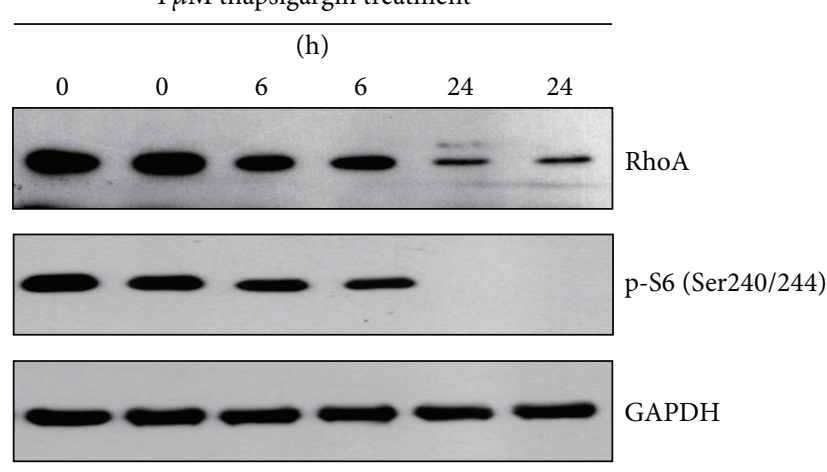

(a)

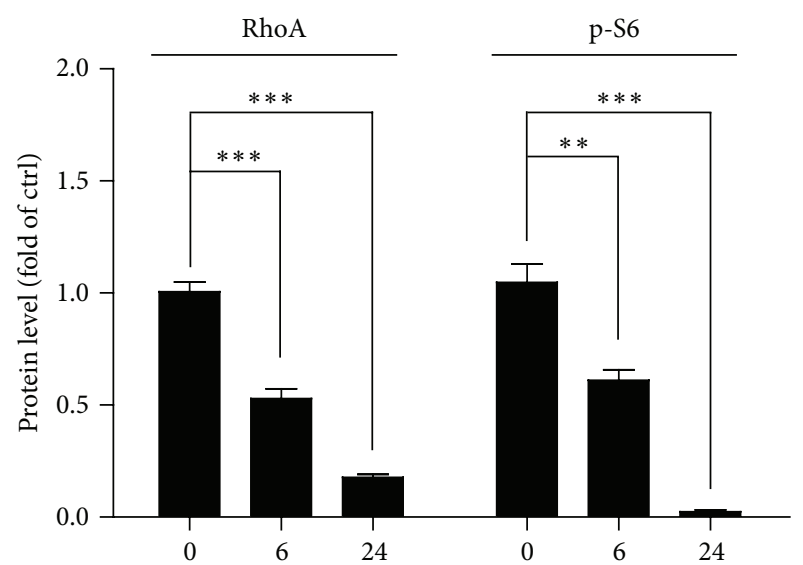

(h)

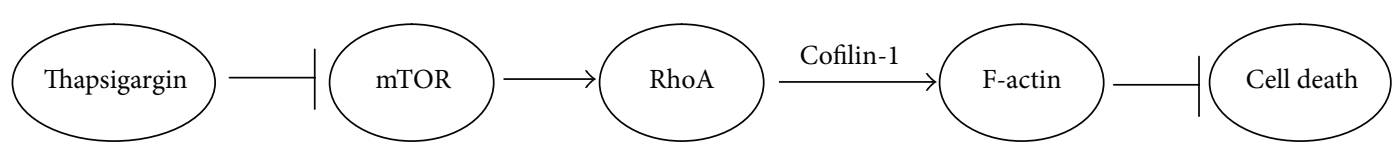

(c)

Figure 4: Thapsigargin impairs cytoskeletal dynamics via mTOR-RhoA pathways in A549 cells. The changes in the protein levels of RhoA and pS6 (S240/244) by thapsigargin treatment ( $1 \mu \mathrm{M}$ for 6 and $24 \mathrm{~h}$ ) in A549 cells were shown by Western blots (a) and histograms (b). Results are averages of four independent experiments. Data represent mean \pm SEM. ${ }^{* *} P<0.01,{ }^{* * *} P<0.001$. (c) Schematic representation highlighting the models of thapsigargin inducing cell apoptosis by impairing actin cytoskeletons in A549 cells. Thapsigargin may induce cell death in A549 cells, by disrupting the actin cytoskeleton organizations, which is mediated by inhibiting mTOR-RhoA-Cofilin-1 pathways.

to the delay effect of thapsigargin on regulator molecules of cytoskeletons. Taken together, our findings suggest that thapsigargin treatment may impair the balance of cytoskeletal dynamics, depolymerizing actin fibers, and inhibiting actin reorganizations.

\subsection{Thapsigargin Impairs Cytoskeletal Dynamics via mTOR-} RhoA Pathways in A549 Cells. To study the molecular mechanism of thapsigargin's effect on cytoskeleton dynamics, the protein levels of mTORC1 indicators and downstream factor RhoA were examined. Our results reveal that the protein levels of pS6 (Ser240/244), a well-known indicator of mTORC1 activity, are reduced to $61.1 \%(1 \mu \mathrm{M}$ for $6 \mathrm{~h})$ and non-detectable $(1 \mu \mathrm{M}$ for $24 \mathrm{~h})$ after thapsigargin treatment (Figures 4(a) and 4(b)). Subsequently, the protein levels of RhoA, a key regulator of actin cytoskeletal dynamics, are also reduced to $52.9 \%(1 \mu \mathrm{M}$ for $6 \mathrm{~h})$ and $17.7 \%(1 \mu \mathrm{M}$ for $24 \mathrm{~h}$ ) after thapsigargin treatment (Figures $4(\mathrm{a})$ and $4(\mathrm{~b})$ ). The reduction of RhoA protein levels is consistent with previous reduction of signals of RhoA immunostaining after thapsigargin treatment (Figure 2). These results indicated that thapsigargin may impair cytoskeletal dynamics through mTOR-RhoA pathways in A549 cells.

\section{Discussion}

Cancer progression is a multistep process that enables tumor cells to disperse to points far from a given primary tumor mass, and this often leads to metastasis. Cell movement through tissue thus plays a crucial and primary role in cancer progression. This process requires a series of distinct but concerted biological events in which the actin cytoskeleton plays essential roles $[5,15]$. In decades, our understanding of the molecules involved in regulating actin cytoskeletal dynamics has increased. Thapsigargin has been reported to induce cell death in several tumor cell lines, by either increasing the store-mediated calcium entry or ER stress [16-18]. It has been reported that thapsigargin treatment rapidly induce a sustained increase in calcium concentration and DNA fragmentation and induce cell death by altering cell morphology or activating apoptotic pathways [14, 19]. In the present study, we demonstrate that thapsigargin, a specific irreversible inhibitor of ER calcium-ATPase, induces cell death by impairing the cytoskeletal dynamics and actin organizations in A549 human lung adenocarcinoma cell line. This process may be mediated by the mTOR-RhoA-Cofilin-1 pathways, because thapsigargin treatment may dramatically inhibit mTORC1 activity, and reduce RhoA proteins and attenuating Cofilin-1 phosphorylations (Figure 4(c)). mTOR is a central controller of cell proliferation, growth, and survival and functions in cells at least as two complexes, mTORC1 and mTORC2. It has been reported that mTOR pathways regulate tumor cell migration and cancer metastasis. For example, rapamycin suppresses IGF-1 stimulated Factin reorganization and migration in various tumor cell lines by inhibiting mTORC1 activity. Rapamycin may also inhibit F-actin reorganization and cell motility by downregulation of RhoA protein expression and activity $[20,21]$. Our findings highlight an important role of calcium entry in cytoskeleton 
organization and apoptosis and set a stage to the clinical treatment of tumor cell metastasis.

The actin cytoskeleton functions in the generation and maintenance of cell morphology and polarity, in endocytosis and intracellular trafficking, contractility, motility, and cell division. The assembly and disassembly of actin filaments, as well as their organization into functional higher-order networks, are regulated by several extracellular and intracellular signalings $[8,22]$. Thapsigargin activates of calcium entry following the depletion of intracellular calcium stores and couples with cytoskeleton organizations. For example, thapsigargin has been reported to induce actin depolymerization and produce a net decrease in F-actin content in human monocytic cells. In turn, prolonged treatment of inhibitors of actin polymerisation abolishes calcium entry by $50 \%$ in human platelets [14]. Thus, the coupling of calcium signaling and actin cytoskeletal dynamics need to be further consolidated. Our findings reveal that ectopic opening of calcium influx by thapsigargin may disrupt the organizations of actin cytoskeletons, which may help to understand the relationship of calcium signaling and cytoskeletal dynamics.

It is well established that increased F-actin may promote cell longevity, whereas decreased actin turnover seems to trigger cell death [7]. The actin regulatory protein Cofilin-1 has been shown to have a key role in the apoptotic process. Cofilin-1 is a member of the Cofilin-1/ADF (actin depolymerizing factor) family and regulates actin dynamics by promoting the depolymerization and severing of actin filaments, and regulating the recycling of the resulting monomers. It has been shown that the active (dephosphorylated) form of Cofilin-1 is targeted to mitochondria after initiation of apoptosis. Mitochondrial targeting of Cofilin-1 is sufficient to induce cytochrome c leakage from the mitochondria and strongly induced apoptosis [23]. Moreover, paxillin, a multidomain protein, is one of the key components of for integrin-mediated cytoskeletal reorganization. In tumor cells, paxillin is highly phosphorylated at Tyr118 and recruits other signalling molecules to focal adhesions for tumor metastasis $[7,24]$. In the present study, our findings suggested that thapsigargin treatment may dramatically reduce Cofilin-1 phosphorylations and increase its activity (Figures 3(a) and 3(b)), which contribute to the actin depolymerization and initiation of apoptosis. To clarify how thapsigargin inhibits Cofilin-1 phosphorylations, we focus on the mTOR-RhoA pathways. mTOR has been shown to integrate signals from a variety of extracellular inputs, including growth factors, amino acids, glucose, ATP, and oxygen. mTOR-dependent signaling modulates numerous cellular properties, including cell proliferation, cell motility, and protein translation. Inhibition of mTOR kinase activity by rapamycin impairs mTOR-mediated protein synthesis and activities of the small GTPases (e.g., RhoA), leading to inhibition of F-actin organization and cell motility $[21,25]$. Moreover, the inhibitory effect of rapamycin on expression of RhoA is also observed in other tumor cell lines, including those derived from cervical cancer (HeLa), prostate cancer (PC-3), Ewing sarcoma (Rh1), and glioblastoma (U-373) [26], suggesting that this is not celltype dependent. Here, our results suggested that thapsigargin may also down-regualte mTOR kinase activity and inhibit
RhoA protein level in A549 human lung adenocarcinoma cells. These findings may contribute to the impairment of actin cytoskeletons by thapsigargin. Thus, our work has set up links of calcium influx, mTOR-RhoA pathways, and cytoskeletal dynamics.

\section{Conclusion}

In summary, the present studies reveal that ectopic calcium influx by thapsigargin inhibits mTOR kinase activity and RhoA expressions, thus leading to the increasing of Cofilin1 activity and actin depolymerizations. The impairment of actin cytoskeletal dynamics finally triggers cell death in A549 human lung adenocarcinoma cells. Our work suggests that therapies that specifically target calcium-cytoskeleton signaling molecules may prove useful for the treatment of tumor cell metastasis.

\section{Conflict of Interests}

There is no conflict of interests to declare, and each author certifies that they have no commercial associations that might pose a conflict of interests in connection with this paper.

\section{References}

[1] M. L. Gardel, K. E. Kasza, C. P. Brangwynne, J. Liu, and D. A. Weitz, "Chapter 19 mechanical response of cytoskeletal networks," Methods in Cell Biology, vol. 89, pp. 487-519, 2008.

[2] D. A. Fletcher and R. D. Mullins, "Cell mechanics and the cytoskeleton," Nature, vol. 463, no. 7280, pp. 485-492, 2010.

[3] A. Hall, "The cytoskeleton and cancer," Cancer and Metastasis Reviews, vol. 28, no. 1-2, pp. 5-14, 2009.

[4] T. D. Pollard and J. A. Cooper, "Actin, a central player in cell shape and movement," Science, vol. 326, no. 5957, pp. 1208-1212, 2009.

[5] H. Yamaguchi and J. Condeelis, "Regulation of the actin cytoskeleton in cancer cell migration and invasion," Biochimica et Biophysica Acta, vol. 1773, no. 5, pp. 642-652, 2007.

[6] J.-M. Trifaró, T. Lejen, S. D. Rosé, T. D. Pene, N. D. Barkar, and E. P. Seward, "Pathways that control cortical F-actin dynamics during secretion," Neurochemical Research, vol. 27, no. 11, pp. 1371-1385, 2002.

[7] J. Stricker, T. Falzone, and M. L. Gardel, "Mechanics of the Factin cytoskeleton," Journal of Biomechanics, vol. 43, no. 1, pp. 9-14, 2010.

[8] D. Yamazaki, S. Kurisu, and T. Takenawa, "Regulation of cancer cell motility through actin reorganization," Cancer Science, vol. 96, no. 7, pp. 379-386, 2005.

[9] X.-S. Jiang, C. A. Wassif, P. S. Backlund et al., "Activation of Rho GTPases in Smith-Lemli-Opitz syndrome: pathophysiological and clinical implications," Human Molecular Genetics, vol. 19, no. 7, pp. 1347-1357, 2010.

[10] J. A. Rosado and S. O. Sage, "The actin cytoskeleton in storemediated calcium entry," Journal of Physiology, vol. 526, no. 2, pp. 221-229, 2000.

[11] Y. Wang, M. P. Mattson, and K. Furukawa, "Endoplasmic reticulum calcium release is modulated by actin polymerization," Journal of Neurochemistry, vol. 82, no. 4, pp. 945-952, 2002. 
[12] E. Ferrary, M. Cohen-Tannoudji, G. Pehau-Arnaudet et al., "In vivo, villin is required for $\mathrm{Ca}^{2+}$-dependent $\mathrm{F}$-actin disruption in intestinal brush borders," Journal of Cell Biology, vol. 146, no. 4, pp. 819-829, 1999.

[13] J. A. Rosado and S. O. Sage, "A role for the actin cytoskeleton in the initiation and maintenance of store-mediated calcium entry in human platelets," Trends in Cardiovascular Medicine, vol. 10, no. 8, pp. 327-332, 2000.

[14] S.-Y. Lee, M.-S. Lee, R. P. Cherla, and V. L. Tesh, "Shiga toxin 1 induces apoptosis through the endoplasmic reticulum stress response in human monocytic cells," Cellular Microbiology, vol. 10, no. 3, pp. 770-780, 2008.

[15] M. F. Olson and E. Sahai, "The actin cytoskeleton in cancer cell motility," Clinical and Experimental Metastasis, vol. 26, no. 4, pp. 273-287, 2009.

[16] J. D. Croxtall, Q. Choudhury, J. O. White, and R. J. Flower, "Tamoxifen inhibits the release of arachidonic acid stimulated by thapsigargin in estrogen receptor-negative A549 cells," Biochimica et Biophysica Acta, vol. 1349, no. 3, pp. 275-284, 1997.

[17] J. L. Armstrong, R. Flockhart, G. J. Veal, P. E. Lovat, and C. P. F. Redfern, "Regulation of endoplasmic reticulum stress-induced cell death by ATF4 in neuroectodermal tumor cells," Journal of Biological Chemistry, vol. 285, no. 9, pp. 6091-6100, 2010.

[18] T. De Raedt, Z. Walton, J. L. Yecies et al., "Exploiting cancer cell vulnerabilities to develop a combination therapy for ras-driven tumors," Cancer Cell, vol. 20, no. 3, pp. 400-413, 2011.

[19] I. Földi, A. M. Tóth, Z. Szabó et al., "Proteome-wide study of endoplasmic reticulum stress induced by thapsigargin in $\mathrm{N} 2 \mathrm{a}$ neuroblastoma cells," Neurochemistry International, vol. 62, no. 1, pp. 58-69, 2013.

[20] R. Zoncu, A. Efeyan, and D. M. Sabatini, "MTOR: from growth signal integration to cancer, diabetes and ageing," Nature Reviews Molecular Cell Biology, vol. 12, no. 1, pp. 21-35, 2011.

[21] I. Bracho-Valdés, P. Moreno-Alvarez, I. Valencia-Martínez, E. Robles-Molina, L. Chávez-Vargas, and J. Vázquez-Prado, "MTORC1- and mTORC2-interacting proteins keep their multifunctional partners focused," IUBMB Life, vol. 63, no. 10, pp. 896-914, 2011.

[22] M. Yilmaz and G. Christofori, "EMT, the cytoskeleton, and cancer cell invasion," Cancer and Metastasis Reviews, vol. 28, no. 1-2, pp. 15-33, 2009.

[23] B. W. Bernstein and J. R. Bamburg, "ADF/Cofilin: a functional node in cell biology," Trends in Cell Biology, vol. 20, no. 4, pp. 187-195, 2010.

[24] K. Pallier, A.-M. Houllier, D. Le Corre, A. Cazes, P. LaurentPuig, and H. Blons, "No somatic genetic change in the paxillin gene in nonsmall-cell lung cancer," Molecular Carcinogenesis, vol. 48, no. 7, pp. 581-585, 2009.

[25] H. Zhou and S. Huang, "Role of mTOR signaling in tumor cell motility, invasion and metastasis," Current Protein and Peptide Science, vol. 12, no. 1, pp. 30-42, 2011.

[26] L. Liu, Y. Luo, L. Chen et al., "Rapamycin inhibits cytoskeleton reorganization and cell motility by suppressing RhoA expression and activity," Journal of Biological Chemistry, vol. 285, no. 49, pp. 38362-38373, 2010. 


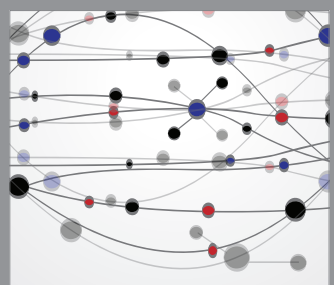

The Scientific World Journal
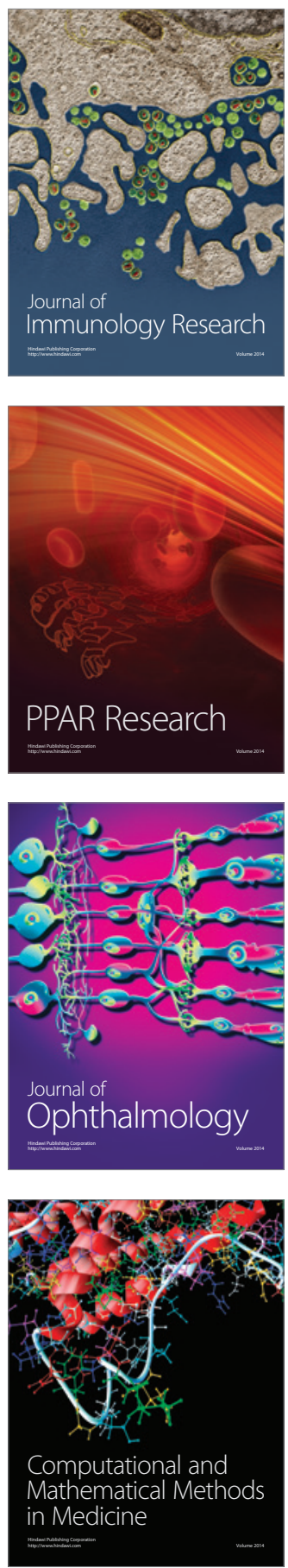

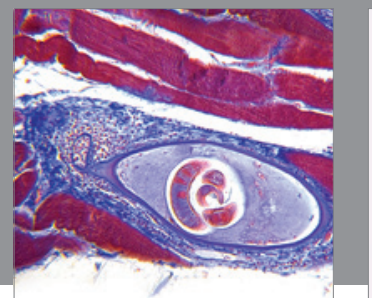

Gastroenterology

Research and Practice
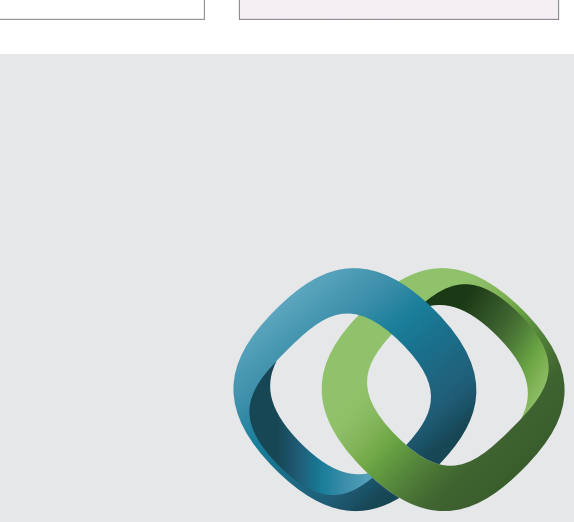

\section{Hindawi}

Submit your manuscripts at

http://www.hindawi.com
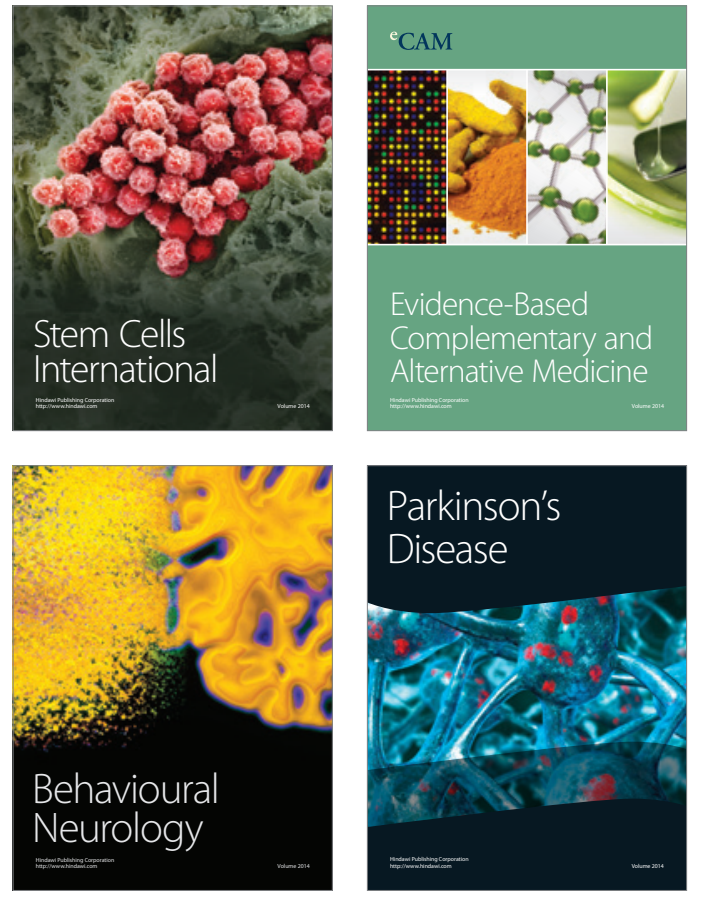
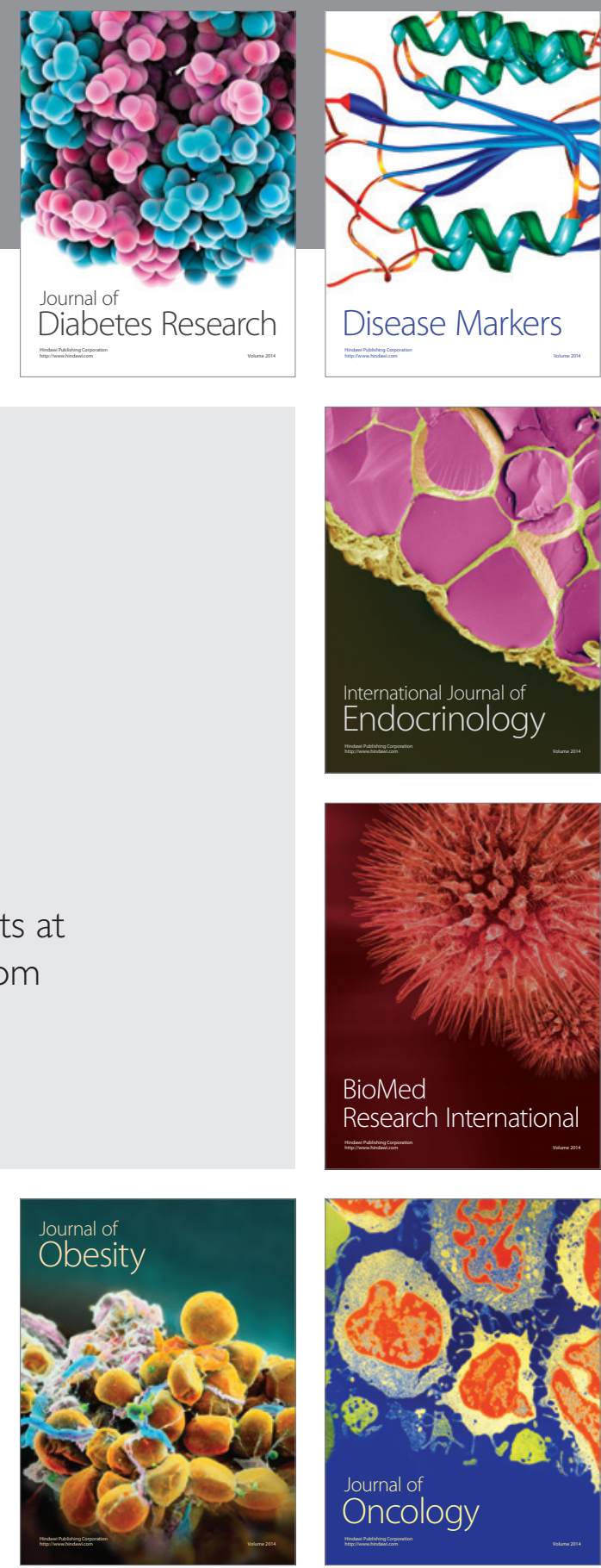

Disease Markers
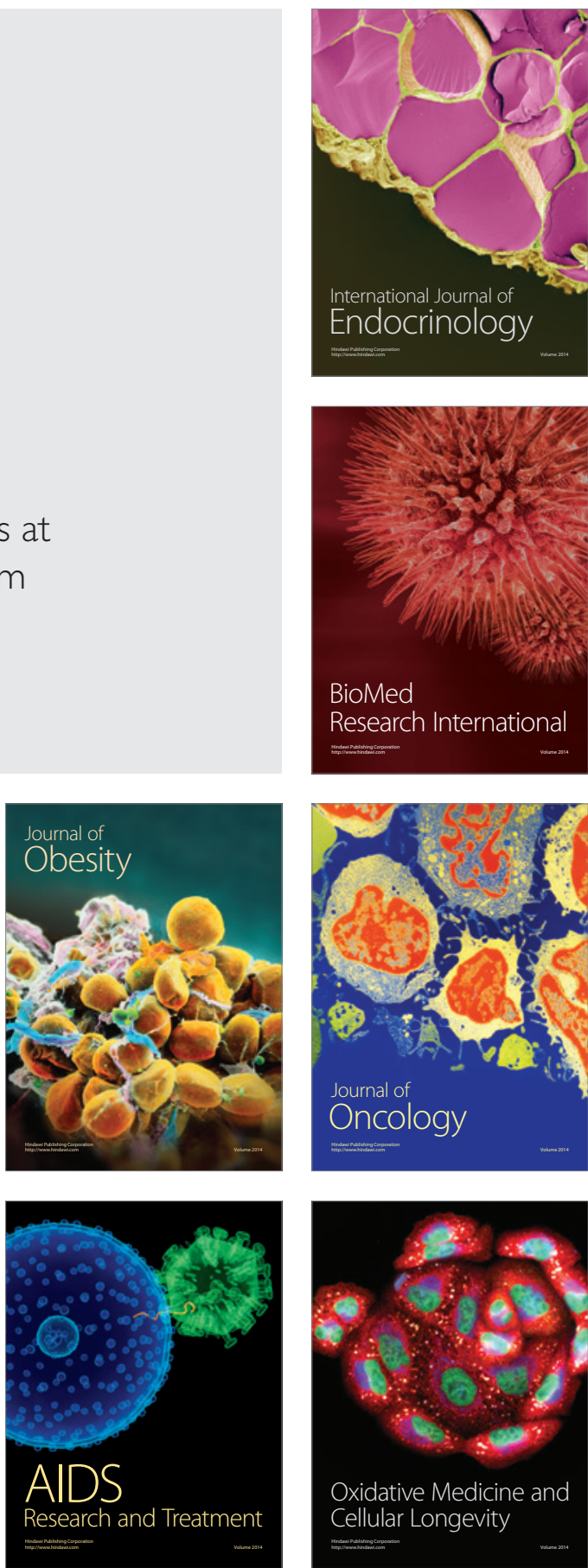